\title{
A new PGAI-NT setup at the NIPS facility of the Budapest Research Reactor
}

\author{
T. Belgya, ${ }^{1 *}$ Z. Kis, ${ }^{1}$ L. Szentmiklósi, ${ }^{1}$ Zs. Kasztovszky, ${ }^{1}$ G. Festa, ${ }^{2}$ L. Andreanelli, ${ }^{2}$ M. P. De Pascale, ${ }^{2}$ \\ A. Pietropaolo, ${ }^{2}$ P. Kudejova, ${ }^{3}$ R. Schulze, ${ }^{3}$ T. Materna, ${ }^{4}$ and the Ancient Charm Collaboration \\ ${ }^{I}$ Institute of Isotopes, Hungarian Academy of Sciences, H-1525 Budapest, P.O. Box 77, Hungary \\ ${ }^{2}$ University of Rome Tor Vergata, 1 Via della Ricerca Scientifica, I-00133 Rome, Italy \\ ${ }^{3}$ University of Cologne, 77 Zülpicherstrasse, D-50937, Cologne, Germany \\ ${ }^{4}$ Institut Laue-Langevin, 6, rue Jules Horowitz, BP 156 - 38042 Grenoble Cedex 9, France
}

(Received July 10, 2008)

Prompt gamma activation analysis (PGAA) is a well known tool for non-destructive bulk elemental analysis of objects. The measured concentrations are only representative of the whole sample if it is homogenous; otherwise it provides only a sort of average composition of the irradiated part. In this latter case one has to scan the sample to obtain the spatial distribution of the elements. To test this idea we have constructed a prompt gamma activation imaging - neutron tomograph (PGAI-NT) setup at the NIPS station of the Budapest Research Reactor, consisting of a high-resolution neutron tomograph and a germanium gamma-spectrometer. The samples are positioned relative to the intersection of the collimated neutron beam and the projection of the gamma-collimator (isocenter) by using an xyz $\omega$-moving table.

\section{Introduction}

Prompt gamma activation analysis (PGAA) is a well known tool for non-destructive bulk elemental analysis of objects. In PGAA the impinging neutron beam hits the sample and excites its atomic nuclei. The excited nucleus decays promptly with a cascade of gamma-rays to the ground state of the daughter nucleus. These gamma-rays are observed with a high resolution germanium gamma-ray detector and the resulting spectrum is collected in a multi-channel analyzer. The prompt gamma-ray spectrum can contain several hundred peaks. The peak energies are characteristic for the emitting atomic nucleus, while their intensities are proportional to the number of atoms in the sample. In this way the concentration of the elements or even isotopes can be determined in the sample. ${ }^{1}$

The neutron source in our case is the 10-MW Budapest Research Reactor. The neutrons are cooled with a cold source and guided to the experimental area with a supermirror guide. ${ }^{2}$ Just before the experimental area the neutrons are divided into two beams with a collimators. The upper one serves the PGAA facility, while the lower beam goes further and serves the NIPS facility. A recent detailed description is given by RÉVAY et al. ${ }^{3}$ The NIPS experimental station can accommodate various measurement configurations such as gammagamma coincidence or a new type of setup, which is the subject of this article.

One of the aims of the EU-FP6 supported "Ancient Charm" (AC) collaboration (http://ancientcharm.neutron-eu.net/ach/) is to develop sophisticated instruments to analyze the internal composition of complex archaeological objects ${ }^{4}$ in order to study various archaeological questions such as production technology, trading paths, and provenance. The simultaneous use of prompt gamma activation imaging (PGAI) and neutron tomography (NT) can nondestructively provide the $3 \mathrm{D}$-distribution of elements in interesting regions of objects. ${ }^{5}$ The NT is a well established method to provide three dimensional imaging of an object, but is not able to determine the elemental distributions. ${ }^{6}$

The PGAI is a new terminology - introduced in the project - for determining the compositions of small volumes while scanning over the sample. This volume is determined by the intersection of the collimated neutron beam and the viewing solid angle of a highly collimated gamma-ray detector. In medical imaging, this kind of intersection is called the isocenter, which is a fixed point in space and is the source of the information. In our case it is a small volume rather than a point; therefore, it is better to call it the isovolume. If a sample is moved around the isovolume we can scan it by acquiring a spectrum at each position. A complete $3 \mathrm{D}$ scan of the object would take a lot of time, however, by using the 3D NT image of the object; one can measure specifically at the interesting regions and determine only their compositions. This combination of the NT with PGAI speeds up considerably the effective scanning of the object.

Furthermore, other non-destructive neutron based methods such as neutron diffraction (ND), neutron resonance capture imaging (NRCI) or X-ray tomography can also be combined to obtain more and complementary information about the object. This is the ultimate idea of the "Ancient Charm" project. ${ }^{4}$ In this article, we focus on the implementation of the PGAI-NT combination.

\footnotetext{
*E-mail: belgya@iki.kfki.hu
} 


\section{The experimental setup}

The setup is an early product of the AC project and many of the collaboration's members contributed (design, hardware and/or software) to its realization.

\section{The PGAI setup}

The schematic drawing of the PGAI-NT setup is shown in Fig. 1. The PGAI/NT setup was designed to accommodate a maximum $10 \mathrm{~cm}$ by $10 \mathrm{~cm}$ object horizontally, but its height can be up to $20 \mathrm{~cm}$. The incident neutron beam had a cross section of $20 \mathrm{~mm}$ by $20 \mathrm{~mm}$, when used for neutron tomography. For PGAI, an adjustable neutron collimator was fabricated from ${ }^{6}$ Li-loaded polymer, which can make a 2-mm wide "pencil" beam with height of 2 to $20 \mathrm{~mm}$. The experimental object was surrounded by a $20 \mathrm{~cm} \times 20 \mathrm{~cm} \times 20 \mathrm{~cm}$ sample chamber made also of ${ }^{6} \mathrm{Li}-$ enriched polymer, which has no bottom. The beam entered this box through a hole slightly larger than the maximum beam diameter on its upstream side and left the box on the opposite side. On this latter side a larger hole enabled neutrons to reach the neutron tomograph. The entrance window of the tomograph was very close to the box. The xyz $\omega$-moving table was placed under the sample chamber. The table can reach almost any point within the chamber. This setup allowed the incoming neutrons to impinge on any desired point on the surface of a sample with dimensions mentioned above. A photograph of the setup is presented in Fig. 2.

The moving table was assembled from standard optical table parts (produced by STANDA Ltd.) at University Tor Vergata, Rome. The gamma collimator was built up from lead bricks on the top of an adjustable table, which also held a high-purity germanium detector (HPGe). The axis of a variable-size lead collimator was put perpendicular to the neutron beam, at about $11 \mathrm{~cm}$ from axis of the neutron collimator. The collimator aperture could be adjusted from $2 \mathrm{~mm} \times 2 \mathrm{~mm}$ to any meaningful rectangular size. A $13 \%$-efficiency Ge detector was placed behind the collimator to view the isovolume of the system. The detector signals were processed with an XIA PIXIE-4 digital signal processor, ${ }^{7}$ which was connected to a data acquisition personal computer.

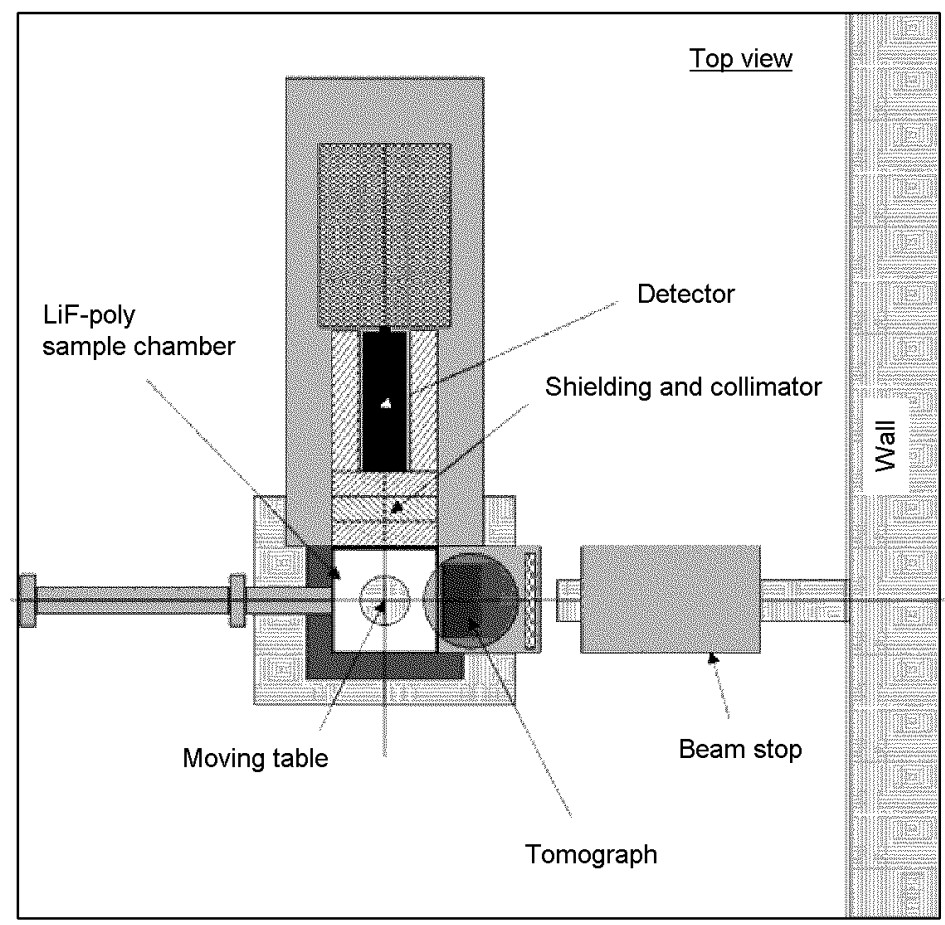

Fig. 1. Drawing of the PGAI-NT setup 


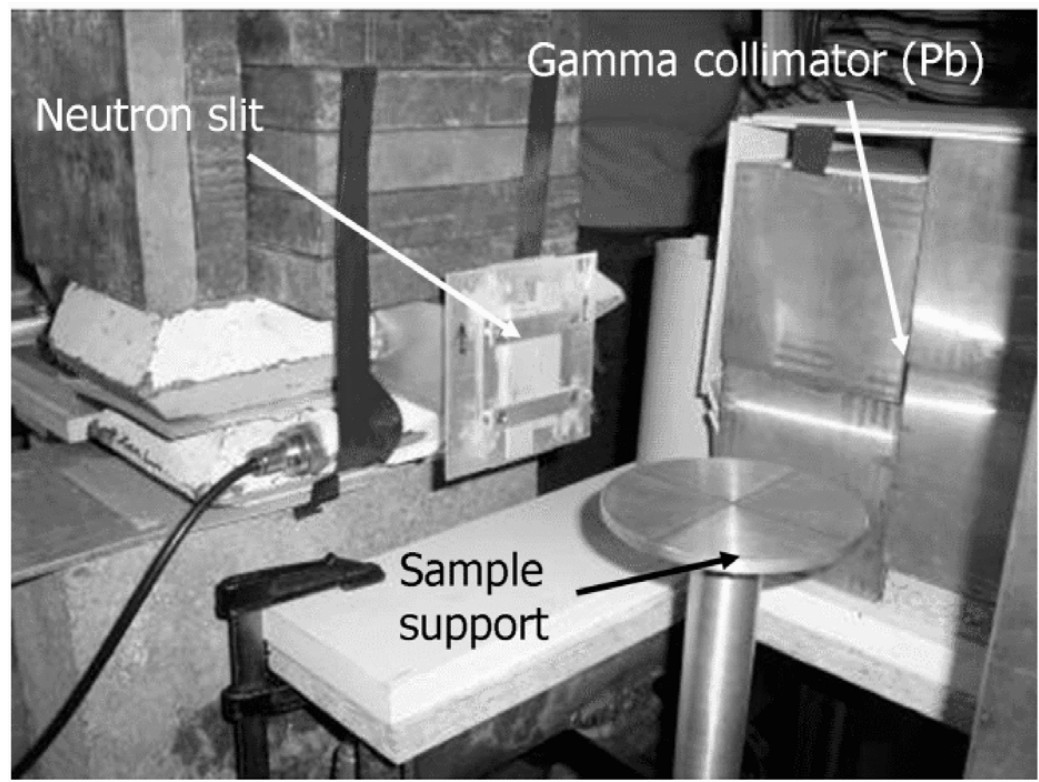

Fig. 2. Photo of the PGAI-NT setup

\section{The neutron tomograph}

The neutron tomograph was provided by the University of Cologne. Its housing was originated from the system formerly used at PSI, Switzerland. The neutron converter scintillator made of ${ }^{6} \mathrm{Li}$ and $\mathrm{ZnS}$ was attached to the housing from inside, and the scintillation light was reflected to the optics by a silver-free mirror. The optics was attached to a high-resolution CCD camera (PCO $1600 \mathrm{BW}$ ) manufactured in Germany. The neutron beam stop placed after the NT was assembled from ${ }^{6} \mathrm{Li}$-enriched polymer, boron and lead.

\section{The data acquisition and analysis software}

An integrated data acquisition system has been developed in the Igor Pro language by the Institute of Isotopes. It is an extension of the gamma acquisition software of XIA written for the PIXIE-4 board. ${ }^{7}$ Now it can control the moving table and can take either gamma spectra, NT images, or both of them in a batch run. The core of the software is the task synchronization: with this the software is capable of operating individual components of the setup in any combination (individually or together). The details (table positions, measurement times, count limits, etc.) of a run can be specified via the so-called batch input table, which should be loaded as a plain text file. Based on the data taken in these runs, the interesting parts of an object can be analyzed. The scan resulted in a large number of prompt spectra, which were analyzed with the spectroscopy software HyperLab 2005b. ${ }^{8}$ We could benefit from its recently added batch evaluation feature. The raw radiography images were analyzed with OCTOPUS tomography reconstruction software developed at University of Ghent. ${ }^{9}$ For complex objects that will be studied later, the coordinates of regions that are to be examined with PGAI will be taken from the NT reconstructions.

\section{Performance and characterization of the setup}

\section{Hardware alignment}

For the precise alignment of the hardware components special measurements were performed with a so called calibration object. It was a $20 \times 20 \times 20 \mathrm{~cm}^{3}$ box with transparent plastic walls that was manufactured with high precision in terms of perpendicularity and size was substituted for the ${ }^{6} \mathrm{Li}$ box. First the beam direction was identified using two arrow shaped $\mathrm{Cd}$ pieces. Their arrow tips were placed at the midpoints of the two opposite sides of the leveled calibration cube. NT images were taken while the box was rotated around its vertical axis until the sharp edges from the $\mathrm{Cd}$ pieces matched up. After this procedure the line that connects the midpoints of the box's two opposite sides were parallel to the neutron beam. The two other faces helped to set the axis of the gamma collimator perpendicular to the neutron beam direction. 


\section{NT resolution}

A thin PVC foil with sharp edges was mounted close to the neutron scintillator, and an image was taken for 10 seconds with binning $1 \times 1$ (no pixel summing). The spatial resolution deduced from the $10 \%-90 \%$ width of the vertical edges is about $330 \mu \mathrm{m}$. Figure 3 shows a vertical projection (of a ROI) of the edge.

\section{Full-neutron beam profile}

The NT device offers an efficient way to get information about the spatial distribution of the beam intensity. The resolution can be as good as the tenth of a millimeter. The beam intensity is proportional to the gray values (with a max. value of $16 \mathrm{~K}$ ) of pixels. The full neutron-beam profile at the NIPS station, measured by the tomograph, is depicted in Fig. 4. The maximum beam size along both axes was $30 \mathrm{~mm}$, although the most useful region of the cross section is about $21 \times 21 \mathrm{~mm}^{2}$. Outside this region the available intensity was less than $20 \%$ of the maximum value. It is clearly visible that the most intense part was not in the geometrical middle. For comparison, the aperture of the ${ }^{6} \mathrm{Li}$ neutron collimator was $21 \times 20 \mathrm{~mm}^{2}$.

\section{Neutron slit profile}

For the PGAI measurements one has to use a highly collimated neutron pencil beam in order to ensure the proper spatial resolution. Originally it was planned to use a cross section of 2 by $2 \mathrm{~mm}$, but it was not feasible with the flux presently available at the NIPS facility $\left(3 \cdot 10^{7} \mathrm{n} \cdot \mathrm{cm}^{-2} \cdot \mathrm{s}^{-1}\right)$. Therefore, the height of the collimator hole was extended to $20 \mathrm{~mm}$, meaning that only elongated objects (or sample parts) can be analyzed.

To use the neutrons most effectively, the middle of the collimator slit was placed close to the maximum value of the beam intensity rather than the geometrical center of the guide. The nominal width of the ${ }^{6} \mathrm{Li}$ collimator was $2 \mathrm{~mm}$, while the measured width (FWHM) of the pencil beam was found to be about $2.3 \mathrm{~mm}$ (Fig. 5).

\section{Gamma slit profile}

To find out the size of the isovolume, the source of the analytical information, the knowledge of gamma collimator's properties are also important. As the transmission of the lead collimator is energy-dependent, besides the careful simulations, its experimental determination was also necessary. This was achieved by moving a ${ }^{152} \mathrm{Eu}$ point-like source in front of the $2-\mathrm{mm}$ wide slit. The relative peak areas of selected peaks were plotted against the displacement from the geometrical center. The results are presented in Fig. 6. The collimation becomes less effective for higher energies; therefore, we used lower energy peaks in the analysis. The total width of the distribution at the FWHM is about 4-6 mm. With these values the estimated size of the isovolume is around $2.3 \times 5 \times 20 \mathrm{~mm}^{3}=230 \mathrm{~mm}^{3}$, for gamma energies of a few hundred $\mathrm{keV}$.

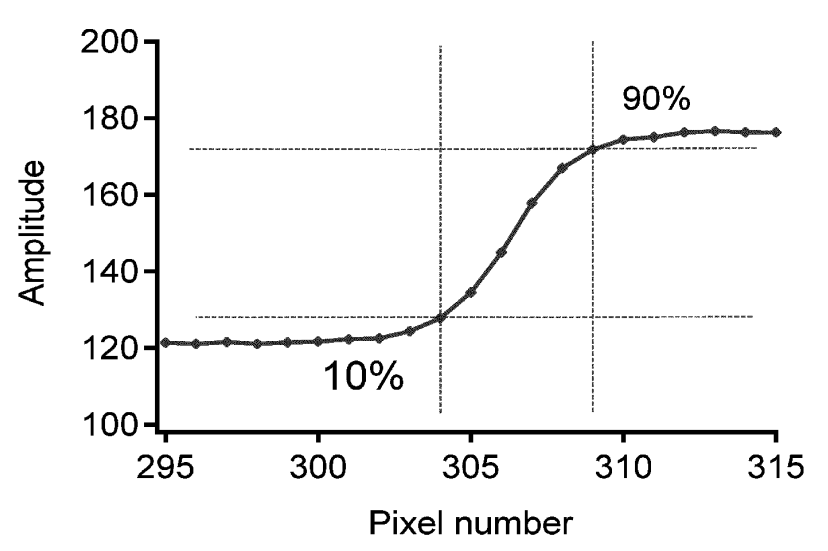

Fig. 3. The neutron flux change around a sharp edge as a function of pixel number. The pixel distance of $10 \%-90 \%$ flux change is about 5 pixels. One pixel is $67 \mu \mathrm{m}$ 


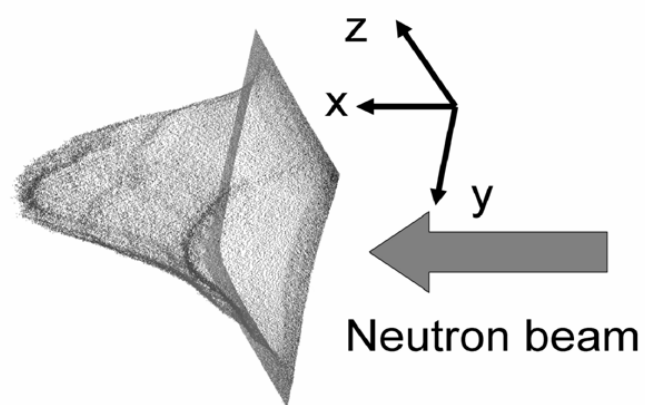

Fig. 4. Full-beam intensity profile at the NIPS station. The value along the $\mathrm{x}$ axis is proportional to the local neutron flux

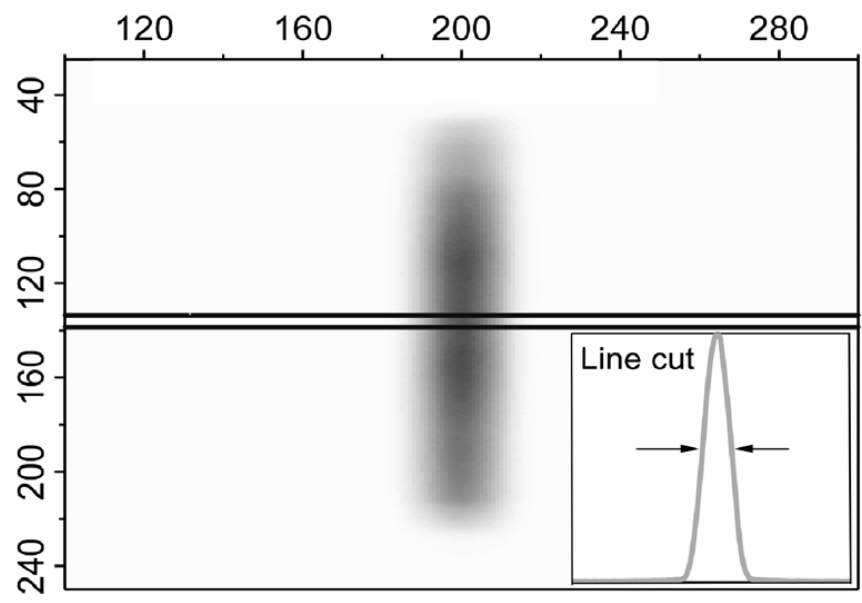

Fig. 5. The neutron pencil beam profile at the NIPS station. The horizontal cut of the matrix is shown in the panel, giving a FWHM of $2.3 \mathrm{~mm}$

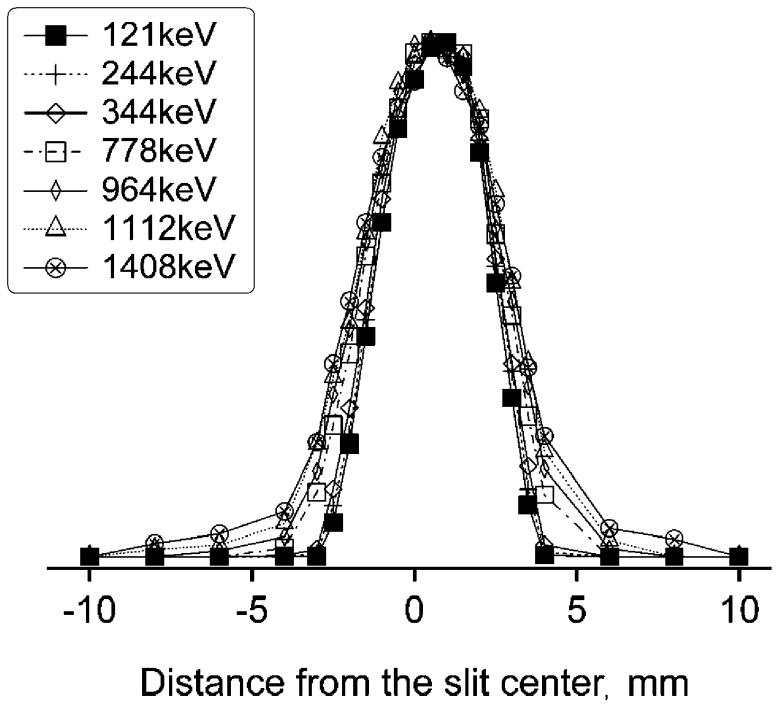

Fig. 6. The transmission of the gamma collimator at different energies 


\section{Conclusions}

A new PGAI-NT facility was built aiming to scrutinize the internal structure and elemental distribution of archeological objects. After, the components of the system were aligned and tested, the basic characteristics of the facility have been determined. This included the measurement of the neutron-beam and gamma-collimator profiles, as well as determining the resolution of the tomograph. Methods were worked out to acquire and process a large number of gamma-spectra.

Efforts to combine the complementary features of neutron tomography and PGAI could lead to an efficient method for investigating valuable cultural heritage objects.

The work was financially supported by the EU FP 6 ANCIENT CHARM Project, Contract No. 15311 and the NAP VENEUS05 Project, Contract No. OMFB-00648/2005.

\section{References}

1. G. L. MoLnÁR, Handbook of Prompt Gamma Activation Analysis with Neutron Beams, Vol. 1, Kluwer Academic Publisher Dordrecht, Boston, London, Budapest, 2004, p. 423.

2. L. Rosta, L. Cser, Z. Révay, Appl. Phys. A-Mater. Sci. Process., 74 (2002) S292.

3. Z. Révay, T. Belgya, Z. Kasztovszky, J. L. Weil, G. L. MolnÁR, Nucl. Instr. Meth. Phys. Res., B213 (2004) 385.

4. G. GoRINI, and the Ancient Charm Collaboration, NuovoCimento, C30 (2007) 47.

5. Z. KASZTOvszky, T. Belgya, Archeometriai Mühely, 2 (2006) 16 (in Hungarian).

6. P. Kudejova, J. Ciyek, R. Schulze, J. Jolie, B. Schillinger, K. Lorenz, M. Mühlbauer, B. Masschaele, M. Dierick, J. VlassenBroeck, Notizario Neutroni E Luce Di Sincrotrone, $12(2007) 6$.

7. Xia Llc, 31057 Genstar Rd., Hayward Ca 94544, USA. http://Www.Xia.Com.

8. A. Simonits, J. ÖstöR, S. KÁlvin, B. FAzeKas, J. Radional. Nucl. Chem., 257 (2003) 589, http://www.hlabsoft.com.

9. Xraylab, Institute for Nuclear Sciences (Inw), Proeftuinstraat 86, 9000 Ghent, Belgium, http://Ssf.Ugent.Be/Linac/Octopus/. 